\title{
A comunicação de notícias difíceis pelos enfermeiros nos cuidados paliativos oncológico pediátricos: uma revisão integrativa
}

The communication of difficult news by nurses in pediatric cancer palliative care: an integrative review

\section{La comunicación de noticias difíciles por parte de las enfermeras en cuidados paliativos del cáncer pediátrico: una revisión integradora}

\section{Resumo}

$\mathrm{O}$ ato de cuidar de uma criança com câncer requer um olhar diferenciado na comunicação de notícias difíceis nos cuidados paliativos oncológico pediátricos. Os objetivos da pesquisa foram: mapear nas produções científicas as estratégias utilizadas pelos enfermeiros na comunicação de notícias difíceis nos cuidados paliativos oncológico pediátrico; descrever e analisar com base nos níveis de evidência as estratégias utilizadas na comunicação de notícias difíceis nos cuidados paliativos oncológico pediátrico. Trata-se de uma revisão integrativa, tendo como pergunta PICO: Quais estratégias são aplicadas pelos enfermeiros na comunicação de notícias difíceis nos cuidados paliativos oncológico pediátrico? Buscou-se nas bases de dados LILACS, CINAHL, Scopus e MEDLINE, a partir dos descritores: enfermagem pediátrica, oncologia, comunicação. Dos resultados, foram construídas 2 categorias acerca das estratégias utilizadas pelo Enfermeiro para a comunicação de notícias difíceis. Em sua maioria, os estudos pertenciam a categoria denominada estratégias humanitárias, que apresentou estratégias como: apoio, diálogo empático, escuta ativa e respeito. A segunda categoria denominada estratégias científicas, apresentou estratégias como: uso de protocolos de comunicações e equipe multiprofissional. São através destas estratégias de cuidado que os enfermeiros fortalecem o vínculo com os familiares e a criança. Os achados evidenciaram uma carência de publicações acerca do tema, bem como ausência de artigos com força de evidência forte.

Palavras-chave: Oncologia pediátrica; Notícias difíceis; Cuidados paliativos; Enfermagem.

\begin{abstract}
The act of caring for a child with cancer requires a different look in the communicating difficult news in pediatric cancer palliative care. The objectives of the research were: to map in scientific productions the strategies used by nurses in the communication of difficult news in pediatric cancer palliative care; to describe and analyze based on the levels of evidence the strategies used in the communication of difficult news in pediatric cancer palliative care. This is an integrative review, with the PICO question: What strategies are applied by nurses in the communication of difficult news in pediatric oncological palliative care? LILACS, CINAHL, Scopus and MEDLINE databases were searched from the descriptors: pediatric nursing, oncology, communication. From the results, two categories were constructed about the strategies used by nurses to communicate difficult news. Most of the studies belonged to the category called humanitarian strategies, which presented strategies such as: support, empathic dialogue, active
\end{abstract}


listening and respect. The second category called scientific strategies presented strategies such as: use of communications protocols and multidisciplinary team. It is through these care strategies that nurses strengthen the bond with family members and the child. The findings showed a lack of publications on the subject, as well as the absence of articles with strong evidence.

Keywords: Pediatric oncology; Difficult news; Palliative care; Nursing.

\section{Resumen}

El acto de cuidar a un niño con cáncer requiere una mirada diferente a la hora de comunicar noticias difíciles en cuidados paliativos de cáncer pediátricos. Los objetivos de la investigación fueron: mapear en producciones científicas las estrategias utilizadas por los enfermeros en la comunicación de noticias difíciles en cuidados paliativos de cáncer pediátrico; describir y analizar con base en los niveles de evidencia las estrategias utilizadas en la comunicación de noticias difíciles en cuidados paliativos de cáncer pediátrico. Se trata de una revisión integradora, con la pregunta PICO: ¿Qué estrategias aplican los enfermeros en la comunicación de noticias difíciles en los cuidados paliativos oncológicos pediátricos? Se realizaron búsquedas en las bases de datos LILACS, CINAHL, Scopus y MEDLINE a partir de los descriptores: enfermería pediátrica, oncología, comunicación. A partir de los resultados, se construyeron dos categorías sobre las estrategias utilizadas por los enfermeros para comunicar noticias difíciles. La mayoría de los estudios pertenecían a la categoría denominada estrategias humanitarias, que presentaba estrategias como: apoyo, diálogo empático, escucha activa y respeto. La segunda categoría denominada estrategias científicas presentaba estrategias como: uso de protocolos de comunicación y equipo multidisciplinario. Es a través de estas estrategias de cuidado que las enfermeras fortalecen el vínculo con los miembros de la familia y el niño. Los hallazgos mostraron una falta de publicaciones sobre el tema, así como la ausencia de artículos con evidencia sólida.

Palabras clave: Oncología pediátrica; Noticias difíciles; Cuidados paliativos; Enfermería.

\section{Introdução}

O câncer na criança corresponde a um grupo de várias doenças que têm em comum a proliferação descontrolada de células anormais e que pode ocorrer em qualquer local do organismo (Brasil, 2019). O processo de adoecimento pelo câncer em uma criança afeta as famílias, que vivenciam de modo intenso (Alves et al., 2016; Duarte et al, 2012) esse momento.

O tratamento do câncer infantil é longo e traumático para a criança, família e profissionais envolvidos (Sousa et al., 2019). Durante o tratamento oncológico, algumas crianças podem não responder à terapêutica, após se esgotarem os recursos atuais para cura (Monteiro et al., 2012). No entanto, as crianças com câncer podem se beneficiar dos cuidados paliativos na trajetória da doença (Silva et al., 2015). A Organização Mundial de Saúde define cuidados paliativos para crianças como cuidado total ativo do corpo, da mente e do espirito da criança e o apoio a família, que se inicia no diagnóstico da doença com outros tratamentos que podem prolongar a vida, como a quimioterapia ou a radioterapia, objetivando sempre a manutenção da qualidade de vida (WHO, 2020).

No tratamento do câncer infantil, a qualidade da interação comunicativa e a recepção de informações sobre a doença, o estado físico da criança, os exames, procedimentos e efeitos colaterais são destacados pelos pais como ponto crucial ao longo do tratamento (Kohlsdorf \& Costa Junior, 2018). O modo de comunicar o diagnóstico e o prognóstico a uma criança e seus familiares influencia de forma muito importante à maneira pela qual vão reagir a ambos (Pacheco \& Goldim, 2019).

Necessária para toda e qualquer interação humana, a comunicação é responsável pela transferência de informações (Amorim et al., 2019). Tendo sua origem no latim, communicare (Lima et al., 2020), compõe o processo básico para a prática das relações humana na transmissão da informação, possibilitando a compreensão de uma pessoa para outra (Santos, 2018).

A inefetividade das comunicações ou a dificuldade em revelar determinadas informações no contexto da saúde evidencia a existência de notícias difíceis (Amorim et al., 2019). Associada à doença grave ou a perdas familiares, as notícias difíceis podem representar um risco à vida, à segurança, ao conforto e à tranquilidade pessoal, familiar e social pelos impactos causados a quem a recebe (Amorim et al., 2019).

Comunicar notícias difíceis não é fácil e requer cuidado. Assim, o profissional enfermeiro permanece o maior tempo em contato com os pacientes, e ao ter uma proximidade com a pessoa doente (Santos et al., 2013) utiliza técnicas e protocolos para comunicação de notícias difíceis, evitando que informações incoerentes de outros profissionais tragam inseguranças e 
conflitos em um momento tão delicado.

A maneira como essa notícia difícil é transmitida, interfere diretamente na relação equipe-paciente; no modo como o indivíduo lida com o diagnóstico; na esperança desenvolvida após a notícia; nos relacionamentos pessoais e familiares; na busca por uma melhor qualidade de vida; e na adesão ao tratamento (Neto et. al., 2017). Justificando assim, o objetivo deste trabalho, visando uma melhor relação profissional-paciente, retorno à comunidade cientifica acerca das estratégias utilizadas e proporcionando um melhor atendimento as crianças e suas famílias.

É importante considerar a necessidade de pesquisas, que delimitem a melhor estratégia para a comunicação de más notícias pela equipe de Enfermagem. Diante disso, este artigo tem como objetivos: mapear nas produções científicas as estratégias utilizadas pelos enfermeiros na comunicação de notícias difíceis nos cuidados paliativos oncológico pediátrico; descrever e analisar com base nos níveis de evidencia as estratégias utilizadas na comunicação de notícias difíceis nos cuidados paliativos oncológico pediátrico.

\section{Metodologia}

Trata-se de uma revisão integrativa de literatura, que é um método que tem como finalidade sintetizar resultados obtidos em pesquisas sobre um tema ou questão, de maneira sistemática, ordenada e abrangente. Foram utilizadas seis etapas para a construção desta pesquisa: 1) identificação do tema e seleção da hipótese ou questão de pesquisa; 2) estabelecimento de critérios de exclusão e inclusão de estudos/amostragem ou busca na literatura; 3) definição das informações a serem extraídas dos estudos selecionados/categorização dos estudos; 4) avaliação dos estudos incluídos na pesquisa; 5) interpretação dos resultados; 6) apresentação da revisão/síntese do conhecimento (Mendes et al., 2008).

Para alcançar os objetivos propostos, a revisão foi construída baseada na estratégia PICO, onde cada letra representa um componente da questão norteadora da pesquisa (Camargo et al., 2017). Sendo assim, P: enfermeiros especialistas em oncologia; I: estratégias de comunicação de notícias difíceis; O: cuidados paliativos pediátricos. Nesta revisão integrativa, o terceiro elemento, comparação (C) não foi utilizado, assim dependendo do método de revisão, não se emprega todos os elementos da estratégia PICO (Garcia et al, 2016). Diante disso, a seguinte questão foi construída: Quais estratégias são aplicadas pelos enfermeiros na comunicação de notícias difíceis nos cuidados paliativos oncológico pediátrico?

O processo seguiu as recomendações do Preferred Reporting Items for Systematic Reviews and Meta-Analyses PRISMA (Galvão et al., 2015), que consiste em um checklist, que contém um fluxograma de quatro etapas (identificação, seleção, elegibilidade e inclusão), podendo ser usado como uma base para relatos de revisões sistemáticas, bem como de outros tipos de pesquisa, particularmente avaliações de intervenções (Galvão et al., 2015).

Foram utilizados os seguintes critérios de inclusão: pesquisas que apresentasse a temática referente às estratégias utilizadas pela enfermagem para a comunicação de notícias difíceis na oncologia pediátrica, que contemplassem os objetivos do estudo, com disponibilidade de texto on-line na íntegra e gratuitamente para download. Os critérios de exclusão utilizados foram: artigos que não se encaixavam na referida temática. Para essa pesquisa foi delimitado o recorte temporal de 2010 à 2019, a fim de discutir sobre as evidências científicas nos últimos nove anos.

A coleta de dados foi realizada nos meses de março a junho de 2019, onde foi feita uma busca das produções científicas nas bases de dados eletrônicas Literatura Latino-Americana e do Caribe em Ciências da Saúde (LILACS), Medical Literature Analysis and Retrieval System Online (MEDLINE), Cumulative Index to Nursing and Allied Health Literature (CINAHL), Scientific Electronic Library Online (ScieELO) e Scopus. Utilizou-se na pesquisa a estratégia de busca com os descritores em Ciências da Saúde (DeCS) e descritores não oficiais: Enfermagem pediátrica, oncologia, comunicação, pediatria, oncologia, Enfermagem e cuidados paliativos, nos idiomas português e inglês. Foram realizadas as seguintes estratégias de busca: Enfermagem OR Enfermagem pediátrica OR Pediatria AND Oncologia OR Cuidados Paliativos AND 
Comunicação; Nursing OR Pediatric Nursing AND Oncology OR Palliative Care AND Communication. A busca foi realizada pelo acesso online nas bases de dados selecionadas, de forma independente por dois revisores com a finalidade de diminuir possível viés de verificação dos estudos.

$\mathrm{Na}$ busca das produções científicas, a partir das estratégias adotas, foram encontrados 198 artigos (74 no MEDLINE; 80 na CINAHL; 36 na Scopus e 8 na LILACS), que posteriormente foram lidos os títulos e resumos, dos quais foram selecionados 32 para leitura na íntegra, excluindo-se 156 artigos por não apresentarem em conformidade com os critérios de inclusão, bem como 10 artigos duplicados. Após leitura dos 32 artigos na íntegra foram selecionadas 09 publicações científicas de acordo com a temática estabelecida. O fluxograma de identificação do processo de seleção dos estudos é demonstrado na Figura 1.

Figura 1: Fluxograma de identificação do processo de seleção dos estudos selecionados da revisão integrativa, elaborado a partir da recomendação PRISMA.
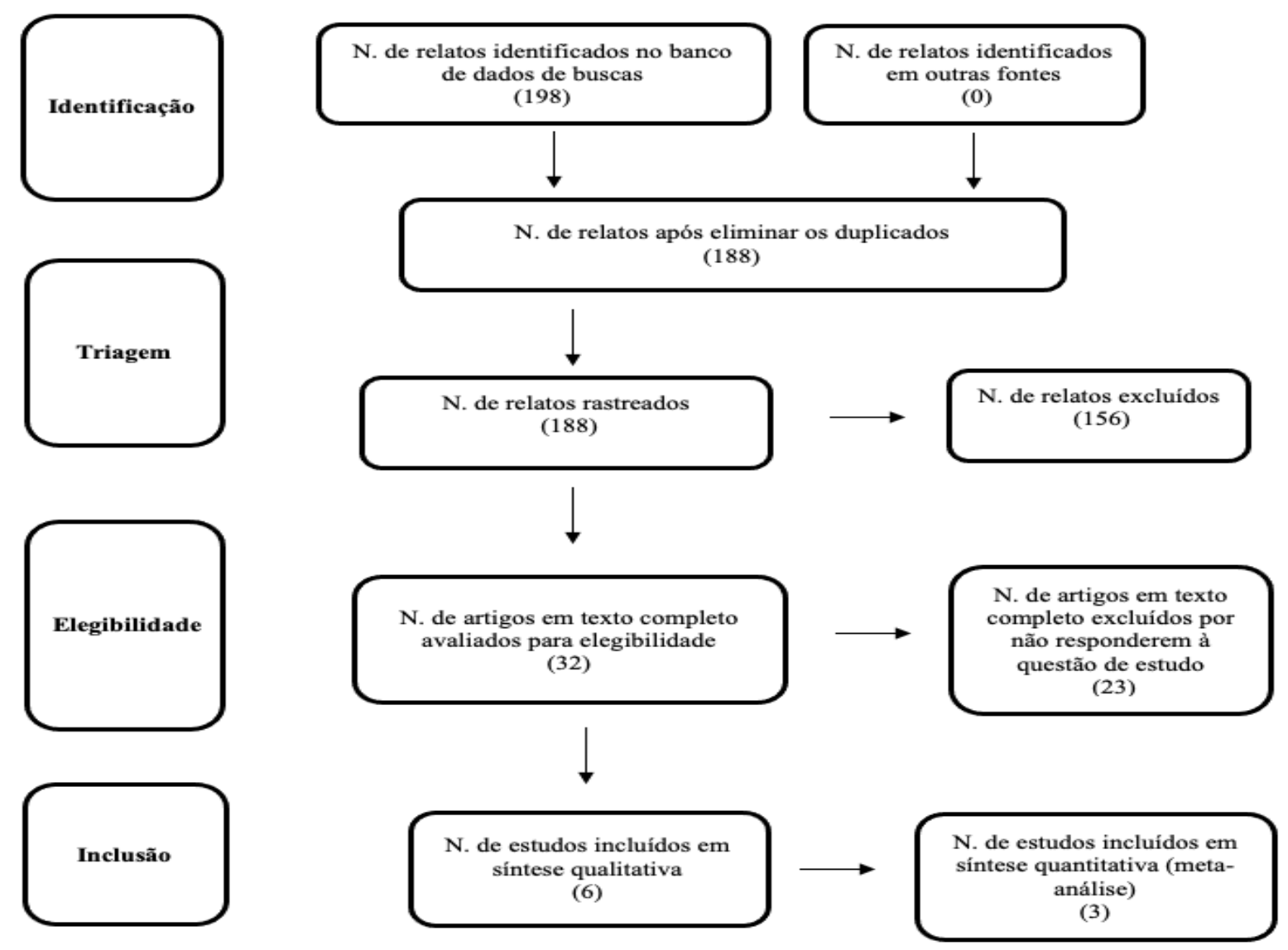

Fonte: Autores (2019).

As publicações selecionadas foram catalogadas e organizadas no software Microsoft Excel, em um instrumento de criação própria, contendo as seguintes informações: título do artigo, periódico, autores, país de origem, ano de publicação, área da saúde, temática, fator de impacto, categoria profissional, base de dados, idioma, tipo de artigo/abordagem, metodologia, aspectos éticos, coleta de dados e tipo de análise. Com a finalidade de facilitar a apresentação dos resultados, cada artigo selecionado recebeu um código de identificação (Z1 à Z9), que serviu de identificação ao longo da análise.

A análise estatística descritiva foi realizada a partir da distribuição de frequência em números absolutos (n) e da 
frequência relativa (\%), disposto em tabela. Após o mapeamento dos artigos, aplicou-se análise temática de conteúdo e a sistematização temático-categorial do corpus da produção científica.

Para classificação dos níveis de evidência, foram considerados sete níveis segundo a classificação de Melnyk e Fineout Overholt (2011), conforme apresentado no Quadro 1.

Quadro 1: Níveis de Evidência segundo Classificação de Melnyk e Fineout-Overholt (2011).

\begin{tabular}{|c|l|}
\hline NE* $^{*}$ & TIPOS DE ESTUDOS \\
\hline I & $\begin{array}{l}\text { Evidência proveniente de revisões sistemáticas ou metanálises de todos os ensaios clínicos randomizados controlados } \\
\text { relevantes ou oriundas de diretrizes clínicas baseadas em revisões sistemáticas de ensaios clínicos randomizados } \\
\text { controlados. }\end{array}$ \\
\hline II & Evidência derivada de pelo menos um ensaio clínico randomizado controlado bem delineado. \\
\hline III & Evidência obtida de ensaios clínicos bem delineados sem randomização. \\
\hline IV & Evidência proveniente de estudo de caso controle ou estudo de coorte bem delineado \\
\hline V & Evidência proveniente de revisões sistemáticas de estudos qualitativos e descritivos \\
\hline VI & Evidência derivada de um estudo descritivo ou qualitativo \\
\hline VII & Evidência oriunda da opinião de autoridades e/ou relatórios de comitês de especialistas \\
\hline
\end{tabular}

NE* - Nível de Evidência. Fonte: Autores (2019).

Em relação aos aspectos éticos e legais, por se tratar de um estudo com metodologia baseada em uma pesquisa bibliográfica somado ao fato que não houve conflito de interesses, não houve necessidade da submissão desta revisão integrativa ao Comitê de Ética em Pesquisa.

\section{Resultados}

Esta revisão é composta por 9 estudos selecionados a partir da leitura minuciosa das produções científicas. O Quadro 2 apresentam os resultados separados pelo número de codificação, a base de dados, o ano de publicação, país, o periódico e o título da produção científica.

Quadro 2: Artigos selecionados separado por codificação, base de dados, ano de publicação, país, periódico, título.

\begin{tabular}{|c|c|c|c|c|c|}
\hline CÓDIGO & $\begin{array}{c}\text { BASE DE } \\
\text { DADOS }\end{array}$ & ANO & PAÍS & PERIÓDICO & \multicolumn{1}{|c|}{ TÍTULO } \\
\hline Z1 & CINAHL & 2019 & EUA & Nursing Outlook & $\begin{array}{l}\text { Pediatric oncology nurses' perceptions of } \\
\text { prognosis-related communication. }\end{array}$ \\
\hline Z2 & CINAHL & 2010 & EUA & Americam Journal Critical Care & $\begin{array}{l}\text { Pediatric nurse's perceptions of obstacles and } \\
\text { supportive behaviors in end-of-life care. }\end{array}$ \\
\hline Z3 & LILACS & 2013 & BRASIL & Revista Enfermagem UERJ & Cuidados paliativos à criança com câncer. \\
\hline Z4 & LILACS & 2013 & BRASIL & $\begin{array}{l}\text { Revista Latino-Americana de } \\
\text { Enfermagem }\end{array}$ & $\begin{array}{l}\text { Importância da comunicação nos cuidados } \\
\text { paliativos em oncologia pediátrica: enfoque na } \\
\text { teoria humanística de enfermagem. }\end{array}$ \\
\hline
\end{tabular}


Research, Society and Development, v. 10, n. 10, e335101018788, 2021

(CC BY 4.0) | ISSN 2525-3409 | DOI: http://dx.doi.org/10.33448/rsd-v10i10.18788

\begin{tabular}{|c|l|l|l|l|l|}
\hline Z5 & MEDLINE & 2014 & EUA & $\begin{array}{l}\text { Journal of Pediatric Oncology } \\
\text { Nursing }\end{array}$ & $\begin{array}{l}\text { Novice nurse's experiences with palliative and } \\
\text { end-of-life communication. }\end{array}$ \\
\hline Z6 & MEDLINE & 2015 & EUA & Pediatric Blood Cancer & $\begin{array}{l}\text { Palliative care as a standard of care in pediatric } \\
\text { oncology. }\end{array}$ \\
\hline Z7 & MEDLINE & 2010 & EUA & Seminars in Oncology Nursing & $\begin{array}{l}\text { Pediatric palliative care in childhood cancer } \\
\text { nursing: from diagnosis to cure or end of life. }\end{array}$ \\
\hline Z8 & MEDLINE & 2012 & EUA & $\begin{array}{l}\text { Clinical Medicine Insights: } \\
\text { Pediatrics }\end{array}$ & $\begin{array}{l}\text { The nursing dimension of providing palliative } \\
\text { care to children and adolescents with cancer. }\end{array}$ \\
\hline Z9 & Scopus & 2013 & TURQUIA & $\begin{array}{l}\text { Asian Pacific Journal of Cancer } \\
\text { Exploring communication difficulties in pediatric }\end{array}$ \\
& & & Prevention & hematology: oncology nurses. \\
\hline
\end{tabular}

Fonte: Autores (2019).

Dos 9 artigos selecionados estão indexados, em sua maioria, na base de dados MEDLINE com 4 (44,44\%) artigos totais. Seguido de: 2 (22,22\%) artigos na LILACS, 2 (22,22\%) artigos na CINAHL e $1(11,11 \%)$ artigo na Scopus. Quanto ao ano de publicação, em ordem de maior para menor frequência, 3 (33,33\%) em 2013, 2 (22,22\%) em 2012, em 2010, 2014, 2015 e 2019 tendo $1(11,11 \%)$ publicação em cada ano.

Quanto ao idioma em que os artigos foram publicados, destaca-se o inglês. Com 7 (77,78\%) das publicações, seguido do português, com 2 (22,22\%). Com relação ao país de publicação, temos os Estados Unidos da América com 6 (66,67\%) publicações, seguido do Brasil, com $2(22,22 \%)$ e da Turquia com $1(11,11 \%)$ publicação.

No que se referem à força de evidência (Melnyk \& Fineout-Overholt, 2011) dos artigos, estes foram classificados com maior frequência em evidências moderadas: IV com 6 (66,67\%) publicações neste nível; evidências baixas: V com 2 (22,22\%) publicações e VII com $1(11,11 \%)$ publicação, e estão representados na Tabela 1.

Tabela 1- Classificação dos artigos selecionados nesta revisão, de acordo com o nível de evidência de Melnyk \& FineoutOverholt (2011).

\begin{tabular}{|ccc|}
\hline NÍVEL DE & N & f\% \\
EVIDÊNCIA & & $0 \%$ \\
\hline I & 0 & $0 \%$ \\
II & 0 & $0 \%$ \\
\hline III & 0 & $66,67 \%$ \\
\hline IV & 6 & $22,22 \%$ \\
\hline V & 2 & $0 \%$ \\
VI & 0 & $11,11 \%$ \\
\hline VII & 1 & $\mathbf{1 0 0 \%}$ \\
\hline TOTAL & $\mathbf{9}$ & \\
\hline
\end{tabular}

Fonte: Autores (2019).

Para uma melhor visualização, e interpretação das estratégias utilizadas pelos enfermeiros para a comunicação de notícias difíceis apresentadas nos artigos, optou-se por categorizar as estratégias encontradas em: estratégias humanitárias e estratégias científicas, que são apresentadas no Quadro 3. 
Quadro 3 - Categorias das estratégias utilizadas pelos enfermeiros na comunicação de notícias difíceis, encontradas nos artigos desta revisão.

\begin{tabular}{|c|c|c|c|}
\hline ESTRATÉGIAS & AÇÕES & n* & Artigos \\
\hline \multirow{15}{*}{ Humanitárias } & Apoio, amparo & 9 & $\mathrm{Z1}, \mathrm{Z} 2, \mathrm{Z} 3, \mathrm{Z4}, \mathrm{Z} 5, \mathrm{Z6}, \mathrm{Z7}, \mathrm{Z8}, \mathrm{Z9}$ \\
\hline & Diálogo Empático & 2 & $\mathrm{Z} 4, \mathrm{Z5}$ \\
\hline & Escuta Ativa & 1 & $\mathrm{Z4}$ \\
\hline & Compreensão & 2 & $\mathrm{Z3}, \mathrm{Z5}$ \\
\hline & Carinho & 1 & $\mathrm{Z3}$ \\
\hline & Lúdico ao lidar com a criança & 1 & $\mathrm{Z3}$ \\
\hline & Observação à comunicação não verbal & 2 & Z3, Z4 \\
\hline & Presença física & 1 & $\mathrm{Z3}$ \\
\hline & Gesto de acolhimento & 1 & $\mathrm{Z4}$ \\
\hline & Confiança & 3 & $\mathrm{Z} 1, \mathrm{Z} 4, \mathrm{Z} 5$ \\
\hline & Criança protagonista - comunicar com a criança & 3 & $\mathrm{Z} 1, \mathrm{Z} 2, \mathrm{Z} 4$ \\
\hline & Sinceridade & 5 & Z1, Z4, Z5, Z6, Z8 \\
\hline & Local e Momento certos & 1 & $\mathrm{Z4}$ \\
\hline & Compaixão & 1 & Z6 \\
\hline & Respeito & 1 & $\mathrm{Z} 2$ \\
\hline \multirow{4}{*}{ Científicas } & Equipe Multiprofissional & 3 & Z5, Z6, Z7 \\
\hline & Conhecimento & 3 & $\mathrm{Z1}, \mathrm{Z} 4, \mathrm{Z} 8$ \\
\hline & Educação em Saúde & 1 & $\mathrm{Z} 2$ \\
\hline & Protocolo de Comunicação & 2 & $\mathrm{Z7}, \mathrm{Z8}$ \\
\hline
\end{tabular}

Nota: $\mathrm{n}^{*}$ quantidade de artigos citados. Fonte: Autores (2019).

Conforme os dados, foram encontradas 15 estratégias que se encaixam dentro da categoria humanitária, correspondendo ao total de 78,95\% das estratégias encontradas nesta revisão. Enquanto 4 estratégias foram consideradas pertencentes a categoria científica, correspondendo a $21,05 \%$ das estratégias encontradas nesta revisão.

\section{Discussão}

A pesquisa no âmbito da temática das estratégias utilizadas pelos enfermeiros na comunicação de más notícias em oncologia pediátrica nos cuidados paliativos, no recorte temporal de nove anos, evidencia uma quantidade considerável de publicações em periódicos internacionais das ciências da saúde em relação aos periódicos nacionais. Os dados também 
apontam, que houve uma lacuna nas publicações científicas entre os anos de 2016 à 2018, confirmando a ausência de afeição dos pesquisadores enfermeiros em estudar no âmbito das notícias difíceis nos cuidados paliativos na pediatria.

A comunicação é a base fundamental das relações e habilidades interpessoais, considerado um instrumento de auxilio terapêutico que ancora a prestação do cuidado individualizado em paciente em cuidados paliativos (Galvão et al., 2017). Na perspectiva dos cuidados em saúde, a comunicação é um componente vital, inerente e necessário, para a relação entre os profissionais, os pacientes e suas famílias com a finalidade de promover o cuidado humanizado (Andrade et al., 2021).

No ambiente de cuidado, a comunicação de más notícias faz parte do dia a dia dos profissionais de saúde. A pesquisa de mapeamento literário aponta que os estudos internacionais e nacionais abordam o importante papel que o enfermeiro desempenha na equipe de cuidados paliativos, como desempenhar o papel importante no processo de divulgação de informações relacionadas ao prognóstico (Newman et al., 2019), bem como identificar os sintomas, coordenando os cuidados e garantindo uma boa comunicação entre as crianças e familiares (Docherty, et al., 2012).

Apesar da relevância da comunicação para os cuidados paliativos, na literatura nacional, em particular na área da Enfermagem, há uma lacuna de estudos que retratem o processo de comunicação em pacientes sem prognóstico de cura (França et al., 2013a). A contribuição da Enfermagem no que se refere à comunicação de notícias difíceis é fundamental para garantir uma análise detalhada acerca do prognóstico e formas de tratamento, seja ele curativo ou paliativo (Docherty, et al., 2012) respeitando as diretivas antecipadas de vontade do paciente, que versa de um conjunto de desejos, prévio e expressamente manifestado pelo paciente quando estiver incapacitado de se expressar, livre e autonomamente a sua vontade sobre cuidados e tratamentos (Cogo et al, 2017).

Tratando-se da comunicação de notícias difíceis, sabe-se que o profissional de enfermagem pode atuar como um elo entre os familiares, a criança e toda a equipe no contexto de doença fora de possibilidade de cura. É através do Enfermeiro que a equipe pode identificar uma comunicação que por ventura tenha sido mal sucedida e ter a oportunidade de reparar esta informação, ou mesmo identificar alguma demanda que a criança venha a apresentar quanto ao seu diagnóstico (Weaver et al., 2015).

Neste contexto, a comunicação configura-se como elemento norteador da eficácia das ações de Enfermagem nos cuidados paliativos, tornando-se parte principal do cuidado com quem vivencia a doença. A comunicação eficaz é a base principal para promover uma assistência de Enfermagem de qualidade, e considerada instrumento fundamental para o cuidado integral e humanizado (Newman et al., 2019).

Na perspectiva da comunicação efetiva, isto é, quando aquele que recebe as notícias difíceis sente-se acolhido, mesmo diante do momento vivenciado, pode-se concluir que se fortaleceu o vínculo entre o emissor (profissional) e o receptor (familiar e/ou criança), garantindo que a comunicação seja realizada no modo verbal e não verbal (França et al, 2013b).

As estratégias utilizadas pelos enfermeiros na comunicação das notícias difíceis encontradas nesta revisão foram categorizadas em: estratégias humanitárias e estratégias científicas. Na categoria da estratégia cientifica, a estratégia da comunicação em equipe multiprofissional foi citada em três artigos (Hendricks-Ferguson et al., 2015; Weaver et al., 2015; Foster et al., 2010) em virtude de ser importante para o fortalecimento das informações (Newman et al., 2019).

A comunicação multiprofissional garante a qualidade do atendimento, quando um ou mais profissionais trabalham juntos, visando encontrar a melhor forma de proporcionar um cuidado eficaz (Newman et al., 2019). Desse modo, possibilita uma sensação de conforto aos profissionais enfermeiros, fazendo com que busquem apoio entre membros da equipe multidisciplinar (Hendricks-Ferguson et al., 2015).

Outra estratégia encontrada foi a educação em saúde, citada apenas em um único artigo (Beckstrand et al., 2010), onde foi considerada uma das principais atividades realizadas pelo enfermeiro para crianças, pais e familiares. Desse modo, o enfermeiro promove a educação em saúde a partir do diálogo simples ou palestra sobre os cuidados paliativos. É importante 
salientar, que se tratando de cuidados paliativos, os pais devem ser orientados sobre retirada de terapias curativas não significa render-se a morte ou abandonar as esperanças (Beckstrand et al., 2010).

Ainda referente às estratégias científicas, o uso de protocolos para a comunicação das notícias difíceis foi uma estratégia pouco citada nas publicações, aparecendo no resultado da pesquisa em dois artigos (Foster et al, 2010; Docherty et al., 2012). Diante de um tema onde se faz presente um misto de emoções e receios, o uso de protocolos especializados e validados facilitaria a comunicação das notícias difíceis. Entretanto, os protocolos foram citados como uma forma de guiar os familiares a dar a noticia a outras pessoas (Citak et al., 2013), e não como indicados como ferramentas utilizadas na prática pelo profissional, deixando explícito o quanto as ações subjetivas são presentes no contexto assistencial dos enfermeiros.

Ao se tratar das estratégias humanitárias, em um dos estudos (Foster et al., 2010) os pais de crianças com câncer apontaram que foram especialmente confortados por enfermeiros ao fornecer a presença e o apoio contínuo durante os cuidados paliativos. Entende-se como estratégia de apoio, o sentido de estar presente, e de incentivar e auxiliar os pais nas tomadas de decisões. Outra estratégia humanitária relatada na revisão foi a presença do enfermeiro nos momentos de tristeza da criança, permitindo que a mesma expresse seus sentimentos momentos difíceis e de superação. Cabe ao profissional estar presente, ajudando a criança, pais e familiares a entender o processo de saúde e doença, dentro das condições do prognóstico (Docherty, et al., 2012).

$\mathrm{Na}$ categoria das estratégias humanitárias, a sinceridade foi citada em cinco artigos (Newman et al., 2019; França et al., 2013a; Hendricks-Ferguson et al., 2015; Weaver et al., 2015; Docherty, et al., 2012), ao expressar que por mais que a vivência possa ser dolorosa e desafiadora, o enfrentamento com a verdade é o melhor caminho para fortalecer a confiança e o vínculo formado.

Foi citada em três artigos (Newman et al., 2019; Beckstrand et al., 2010; França et al., 2013a), a importância de colocar a criança, sempre que possível, como protagonista da vivência da doença. Esta estratégia foi explicitada como uma demonstração de respeito, cuidado e compaixão do profissional enfermeiro na assistência à criança, ao valorizar a comunicação como estratégia de humanizar o cuidado, respeitando o tempo e abrindo espaço para a interação durante o processo de finitude (Foster et al., 2010; França et al., 2013a).

Sabe-se que a Enfermagem é tida como a profissão do cuidar, relacionando-se aos sentimentos subjetivos e empáticos. A literatura esclarece que o ato de cuidar (verbal e não verbal), o olhar silencioso ou mesmo a presença física, sugere certo grau de intersubjetividade que assegura o reconhecimento do cuidado por parte daquele que o recebe (França et al., 2013a). Outras estratégias citadas foram a confiança e o respeito, que são demonstradas muitas vezes sem ser preciso a linguagem verbal, demonstrando apoio a quem vivencia esse processo. São através destas estratégias que os enfermeiros fortalecem a relação com os familiares e a criança, tornando-se o elo principal entre a equipe multidisciplinar-paciente-família.

Diante de todo o processo estruturado de revisão não se pode deixar de mencionar sobre a força dos níveis de evidência encontrados nos artigos selecionados. Entre os nove artigos selecionados, seis possuem força de evidência moderada (IV) e três se encontram dentro do nível de evidência baixa (V a VII). Assim, cabe ressaltar, a ausência de produções com níveis de evidência forte nesta pesquisa. Reitera-se a relevância de publicações de estudos atuais com níveis de evidência forte relacionadas as estratégias de cuidado de enfermeiros sobre a comunicação de notícias difíceis em cuidados paliativos na oncologia pediátrica.

\section{Conclusão}

Os enfermeiros saem da graduação para a vida profissional sem a devida preparação acerca de como realizar a comunicação de notícias difíceis, sendo forçados a aprender em sua prática profissional a identificar qual a melhor estratégia a 
ser utilizada. É notória a importância do papel do enfermeiro na equipe de comunicação. Participar ativamente como membro da equipe multidisciplinar na aplicação dos protocolos de notícias difíceis favorece uma comunicação eficaz e humanizada.

Referindo-se aos objetivos desta revisão de mapear, categorizar e analisar as estratégias utilizadas pelos enfermeiros na comunicação de noticias difíceis pode-se afirmar que os mesmos foram alcançados. Entretanto, apesar da importância dos cuidados paliativos, há ainda uma necessidade de se alavancarem pesquisas de aprofundamento desta temática, principalmente no campo da Enfermagem.

Diante da análise quantitativa e qualitativa desta revisão, observou-se uma grande necessidade da publicação de novos artigos, bem como de produções brasileiras atualizadas acerca do tema e com níveis de evidências fortes, visando proporcionar um melhor atendimento as crianças e suas famílias.

\section{Referências}

Alves, K. D. M. C., Comassetto, I., Almeida, T. G. D., Trezza, M. C. S. F., Silva, J. M. D. O., \& Magalhães, A. P. N. D. (2016). A vivência dos pais da criança com câncer na condição de impossibilidade terapêutica. Texto e Contexto Enfermagem, 25(2):e2120014. https://doi.org/10.1590/0104-07072016002120014

Amorim, C. B., Barlem, E. L. D., Mattos, L. M. D., Costa, C. F. S. D., \& Oliveira, S. G. D. (2019). Comunicação de notícias difíceis na atenção básica à saúde: barreiras e facilitadores percebidos por enfermeiras. Revista Gaúcha de Enfermagem, 40:e20190017. https://doi.org/10.1590/1983-1447.2019.20190017

Andrade, C. G. D., Costa, I. C. P., Freire, M. E. M., Dias, T. K. C., França, J. R. F. D. S., \& Costa, S. F. G. D. (2021). Produção científica acerca dos cuidados paliativos e comunicação em periódicos online: revisão de escopo. Revista Brasileira de Enfermagem, 74 (2). https://doi.org/10.1590/0034-7167-2019-0378

Beckstrand, R. L., Rawle, N. L., Callister, L., \& Mandleco, B. L. (2010). Pediatric nurses' perceptions of obstacles and supportive behaviors in end-of-life care. American journal of critical care: an official publication, American Association of Critical-Care Nurses, 19(6), 543-552. https://doi.org/10.4037/ajcc2009497

Brasil. (2019). Ministério da Saúde. Instituto Nacional de Câncer José Alencar Gomes da Silva. Estimativa 2020: incidência de câncer no Brasil. https://www.inca.gov.br/sites/ufu.sti.inca.local/files//media/document//estimativa-2020-incidencia-de-cancer-no-brasil.pdf

Camargo, E. B., Pereira, A. C. E. da S., Gliardi, J. da M., Pereira, D. R., Puga, M. E., da Silva, E. T., \& Elias, F. T. S. (2017). Judicialização da saúde: onde encontrar respostas e como buscar evidências para melhor instruir processos. Cadernos Ibero-Americanos de Direito Sanitário, 6(4), 27-40. https://doi.org/10.17566/ciads.v6i4.410

Citak, E. A., Toruner, E. K., \& Gunes, N. B. (2013). Exploring communication difficulties in pediatric hematology: oncology nurses. Asian Pacific journal of cancer prevention: APJCP, 14(9), 5477-5482. https://doi.org/10.7314/apjcp.2013.14.9.5477

Cogo, S. B., Lunardi, V. L., \& Nietsche, E. A. (2017). Considerações acerca da atuação do enfermeiro na aplicabilidade das diretivas antecipadas de vontade. Enfermagem em Foco, 8(2): 26-30. http://revista.cofen.gov.br/index.php/enfermagem/article/view/1061

Docherty, S. L., Thaxton, C., Allison, C., Barfield, R. C., \& Tamburro, R. F. (2012). The nursing dimension of providing palliative care to children and adolescents with cancer. Clinical Medicine Insights: Pediatrics, 6, 75-88. https://doi.org/10.4137/CMPed.S8208

Duarte, M. D. L. C., Zanini, L. N., \& Nedel, M. N. B. (2012). O cotidiano dos pais de crianças com câncer e hospitalizadas. Revista Gaúcha de Enfermagem, 33(3), 111-118. https://www.scielo.br/pdf/rgenf/v33n3/15.pdf

Foster, T. L., Lafond, D. A., Reggio, C., \& Hinds, P. S. (2010). Pediatric palliative care in childhood cancer nursing: from diagnosis to cure or end of life. Seminars in Oncology Nursing, 26(4), 205-221. https://doi.org/10.1016/j.soncn.2010.08.003

França, J. R. F. D. S., Costa, S. F. G. D., Lopes, M. E. L., Nóbrega, M. M. L. D., \& França, I. S. X. D. (2013a). Importância da comunicação nos cuidados paliativos em oncologia pediátrica: enfoque na teoria humanística de enfermagem. Revista Latino-Americana de Enfermagem, 21 , $780-786$. http://www.scielo.br/pdf/rlae/v21n3/pt_0104-1169-rlae-21-03-0780.pdf

França, J. R. F. S., Costa, S. F. G., Nóbrega, M. M. L., \& Lopes, M. E. L. (2013b). Cuidados paliativos à criança com câncer. Revista Enfermagem UERJ, 21(6), 779-784. https://www.e-publicacoes.uerj.br/index.php/enfermagemuerj/article/view/12283/9562

Galvão, Maria Ireni Zapalowski, Borges, Moema da Silva, \& Pinho, Diana Lúcia Moura. (2017). Comunicação interpessoal com pacientes oncológicos em cuidados paliativos. Revista Baiana de Enfermagem, 31(3), e22290. https://dx.doi.org/10.18471/rbe.v31i3.22290

Galvão, T. F., Pansani, T. D. S. A., \& Harrad, D. (2015). Principais itens para relatar revisões sistemáticas e meta-análises: a recomendação prisma. Epidemiologia e Serviços de Saúde, 24, 335-342.https://www.scielo.br/scielo.php?script=sci_arttext\&pid=S2237-96222015000200335

Garcia, A. K. A., Fonseca, L. F., Aroni, P., \& Galvão, C. M. (2016). Estratégias para o alívio da sede: revisão integrativa da literatura. Revista Brasileira de Enfermagem, 69, 1215-1222. https://doi.org/10.1590/0034-7167-2016-0317

Hendricks-Ferguson, V. L., Sawin, K. J., Montgomery, K., Dupree, C., Phillips-Salimi, C. R., Carr, B., \& Haase, J. E. (2015). Novice nurses' experiences with palliative and end-of-life communication. Journal of pediatric oncology nursing: official journal of the Association of Pediatric Oncology Nurses, 32(4), 240252. https://doi.org/10.1177/1043454214555196 
Research, Society and Development, v. 10, n. 10, e335101018788, 2021

(CC BY 4.0) | ISSN 2525-3409 | DOI: http://dx.doi.org/10.33448/rsd-v10i10.18788

Kohlsdorf, M., \& Costa Junior A. L. (2018). Procedimento de intervenção aplicado a cuidadores e pacientes relacionado à comunicação em consultas de acompanhamento em câncer infantil. Interação em Psicologia. 22 (3):123-38. http://dx.doi.org/10.5380/psi.v22i3.50913

Lima, K. M. D. A., Maia, A. H. N., \& Nascimento, I. R. C. D. (2020). Comunicação de más notícias em cuidados paliativos na oncopediatria. Revista Bioética, 27, 719-727. https://www.scielo.br/j/bioet/a/YhCskTrnwMgP5QSzw4RSzFC/?lang=pt

Melnyk B. M., \& Fineout-Overholt, E. (2011). Making the case for evidence-based practice. In: Melnyk B. M., \& Fineout-Overholt E. Evidence-based practice in nursing \& healthcare. A guide to best practice (2th ed., pp. 3-24). Lippincot Williams \& Wilkins.

Mendes, K. D. S., Silveira, R. C. D. C. P., \& Galvão, C. M. (2008). Revisão integrativa: método de pesquisa para a incorporação de evidências na saúde e na enfermagem. Texto \& contexto-enfermagem, 17, 758-764. http://www.scielo.br/scielo.php?script=sci_arttext\&pid=S0104-07072008000400018

Monteiro, A. C. M., Rodrigues, B. M. R. D., \& Pacheco, S. T. D. A. (2012). O enfermeiro e o cuidar da criança com câncer sem possibilidade de cura atual. Escola Anna Nery, 16, 741-746. https://doi.org/10.1590/S1414-81452012000400014

Neto, L. L. S., Silva, V. L. L., Lima, C. D. C., Moura, H. T. D. M., Gonçalves, A. L. M., Pires, A. P. B., \& Fernandes, V. G. (2017). Habilidade de comunicação da má notícia: o estudante de medicina está preparado? Revista Brasileira de Educação Médica,41, 260-268. http://www.scielo.br/pdf/rbem/v41n2/1981-5271-rbem-41-2-0260.pdf

Newman, A. R., Haglund, K., \& Rodgers, C. C. (2019). Pediatric oncology nurses' perceptions of prognosis-related communication. Nursing Outlook, 67(1), 101-114. https://doi.org/10.1016/j.outlook.2018.11.001

Pacheco C. L., \& Goldim J. R. (2019). Percepções da Equipe Interdisciplinar Sobre Cuidados Paliativos em Oncologia Pediátrica. Revista Bioética, 27(1): 6775. http://revistabioetica.cfm.org.br/index.php/revista_bioetica/article/view/1793/2022

Santos, R. O. (2018). A importância da comunicação no processo de liderança. Revista de Administração em Saúde, 18(72). https://www.cqh.org.br/ojs2.4.8/index.php/ras/article/view/128/171

Santos, Y. S., Jesus, L. C., \& Portella, S. D. C. (2013). A enfermagem e a abordagem da morte infantil: Um estudo de trabalhos qualis A. Revista Enfermagem Contemporânea, 2(1): 112-131. https://www5.bahiana.edu.br/index.php/enfermagem/article/view/252/215

Silva, A. F. D., Issi, H. B., Motta, M. D. G. C. D., \& Botene, D. Z. D. A. (2015). Cuidados paliativos em oncologia pediátrica: percepções, saberes e práticas na perspectiva da equipe multiprofissional. Revista gaúcha de enfermagem, 36, 56-62. https://doi.org/10.1590/1983-1447.2015.02.46299

Sousa, A. D. R. S., Silva, L. F. D., \& Paiva, E. D. (2019). Intervenções de enfermagem nos cuidados paliativos em Oncologia Pediátrica: revisão integrative. Revista Brasileira de Enfermagem, 72, 531-540. https://doi.org/10.1590/0034-7167-2018-0121

Weaver, M. S., Heinze, K. E., Kelly, K. P., Wiener, L., Casey, R. L., Bell, C. J., Wolfe, J., Garee, A. M., Watson, A., \& Hinds, P. S. (2015). Palliative care as a standard of care in pediatric oncology. Pediatric blood \& cancer, 62 (Suppl 5), 829-33. https://doi.org/10.1002/pbc.25695

World Health Organization-WHO. (2020). Palliative Care. Geneva: WHO. http://www.who.int/mediacentre/factsheets/fs402/en/ 